\title{
Energy Conditions for Hamiltonian and Traceable Graphs
}

\author{
$\operatorname{Rao} \mathbf{L i}^{1^{*}}$ \\ ${ }^{1}$ Dept. of mathematical sciences, University of South Carolina Aiken, Aiken, SC 29801, USA
}

\section{Article Info}

Keywords: Energy, Hamiltonian,

Graph, Traceable

2010 AMS: 05C45, $05 C 50$.

Received: 31 August 2018

Accepted: 29 November 2018

Available online: 20 March 2019

\begin{abstract}
A graph is called Hamiltonian (resp. traceable) if the graph has a Hamiltonian cycle (resp. path), a cycle (resp. path) containing all the vertices of the graph. The energy of a graph is defined as the sum of the absolute values of the eigenvalues of the graph. In this note, we present new conditions based on energy for Hamiltonain and traceable graphs.
\end{abstract}

\section{Introduction}

All the graphs considered in this note are undirected graphs without loops or multiple edges. Notation and terminology not defined here follow those in [1]. Let $G$ be a graph of order $n$ with $e$ edges. We use $\delta(G)$ and $\chi(G)$ to denote the minimum degree and the chromatic number of $G$, respectively. The independence number, denoted $\alpha=\alpha(G)$, is defined as the size of the largest independent set in $G$. The eigenvalues $\mu_{1}(G) \geq \mu_{2}(G) \geq \ldots \geq \mu_{n}(G)$ of the adjacency matrix $A(G)$ of $G$ are called the eigenvalues of $G$. We use $S^{+}(G)\left(\right.$ resp. $S^{-}(G)$ ) to denote the sum of the squares of the positive (resp. negative) eigenvalues of $G$. Notice that $S^{+}(G)+S^{-}(G)=2 e(G)$ for a graph $G$. The energy, denoted $\operatorname{Eng}(G)$, of $G$ is defined as $\sum_{i=1}^{n}\left|\mu_{i}(G)\right|$ (see [2]). A cycle $C$ in a graph $G$ is called a Hamiltonian cycle of $G$ if $C$ contains all the vertices of $G$. A graph $G$ is called Hamiltonian if $G$ has a Hamiltonian cycle. A path $P$ in a graph $G$ is called a Hamiltonian path of $G$ if $P$ contains all the vertices of $G$. A graph $G$ is called traceable if $G$ has a Hamiltonian path. In this note, we will present the energy conditions for Hamiltonian and traceable graphs. The results are as follows.

Theorem 1.1. Let $G$ be a $k$-connected $(k \geq 2)$ graph with $n \geq 3$ vertices and e edges. If

$$
\operatorname{Eng}(G) \geq 2 \sqrt{\frac{2 e(\chi-1)(n-k-1)}{\chi}},
$$

then $G$ is Hamiltonian.

Theorem 1.2. Let $G$ be a $k$-connected graph with $n \geq 3$ vertices and e edges. If

$$
\operatorname{Eng}(G) \geq 2 \sqrt{\frac{2 e(\chi-1)(n-k-2)}{\chi}},
$$

then $G$ is traceable or $K_{1,3}$.

\section{Lemmas}

In order to prove Theorem 1.1, we need the following results as our lemmas. Lemma 2.1 below is Theorem 2.3 on Pages 484 in [3].

Lemma 2.1. Let $G$ be a graph. Then

$$
\chi \geq 1+\max \left\{\frac{S^{+}}{S^{-}}, \frac{S^{-}}{S^{+}}\right\} .
$$


Lemma 2.2 below is Theorem 3.14 on Pages 88 and 89 in [4].

Lemma 2.2. Let $G$ be a graph. If the number of eigenvalues of $G$ which are greater than, less than, and equal to zero are $p$, $q$, and $r$, respectively, then

$$
\alpha \leq r+\min \{p, q\},
$$

where $\alpha$ is the independence number of $G$.

\section{Proofs}

Next, we will present proofs for Theorems 1.1 and 1.2. Some ideas from [5] are used in our proofs.

Proof of Theorem 1.1. Let $G$ be a graph satisfying the conditions in Theorem 1.1. Suppose, to the contrary, that $G$ is not Hamiltonian. If $n=3, G$ must be Hamiltonian since $G$ is $k$-connected $(k \geq 2)$. From now on, we assume that $n \geq 4$. Since $G$ is $k$-connected $(k \geq 2), G$ has a cycle. Choose a longest cycle $C$ in $G$ and give an orientation on $C$. Since $G$ is not Hamiltonian, there exists a vertex $u_{0} \in V(G)-V(C)$. By Menger's theorem, we can find $s\left(s \geq k\right.$ ) pairwise disjoint (except for $\left.u_{0}\right)$ paths $P_{1}, P_{2}, \ldots, P_{s}$ between $u_{0}$ and $V(C)$. Let $v_{i}$ be the end vertex of $P_{i}$ on $C$, where $1 \leq i \leq s$. Without loss of generality, we assume that the appearance of $v_{1}, v_{2}, \ldots, v_{s}$ agrees with the orientation of $C$. We use $v_{i}^{+}$to denote the successor of $v_{i}$ along the orientation of $C$, where $1 \leq i \leq s$. Since $C$ is a longest cycle in $G$, we have that $v_{i}^{+} \neq v_{i+1}$, where $1 \leq i \leq s$ and the index $s+1$ is regarded as 1 . Moreover, $S:=\left\{u_{0}, v_{1}^{+}, v_{2}^{+}, \ldots, v_{s}^{+}\right\}$is independent (otherwise $G$ would have cycles which are longer than $C$ ). Then $\alpha \geq s+1 \geq k+1$.

Let $\mu_{1} \geq \mu_{2} \geq \ldots \geq \mu_{p}$ be the $p$ positive eigenvalues of $G$ and let $\mu_{n-q+1} \geq \mu_{n-q+2} \geq \ldots \geq \mu_{n}$ be the $q$ negative eigenvalues of $G$. Then $n-(p+q)$ is the number of eigenvalues of $G$ which are equal to zero. Since $\sum_{i=1}^{p} \mu_{i}+\sum_{i=n-q+1}^{n} \mu_{i}=\operatorname{trace}$ of $A=0, \sum_{i=1}^{p}\left|\mu_{i}\right|=\sum_{i=n-q+1}^{n}\left|\mu_{i}\right|$. Thus we have that

$$
\operatorname{Eng}(G)=2 \sum_{i=1}^{p}\left|\mu_{i}\right|=2 \sum_{i=n-q+1}^{n}\left|\mu_{i}\right| .
$$

From Lemma 2.1, we have that

$$
\chi \geq 1+\frac{S^{+}}{S^{-}}=1+\frac{S^{+}}{2 e-S^{+}}=\frac{2 e}{2 e-S^{+}}, \chi \geq 1+\frac{S^{-}}{S^{+}}=1+\frac{S^{-}}{2 e-S^{-}}=\frac{2 e}{2 e-S^{-}} .
$$

Therefore we further have that

$$
S^{+} \leq \frac{2 e(\chi-1)}{\chi}, S^{-} \leq \frac{2 e(\chi-1)}{\chi} .
$$

From Cauchy-Schwarz inequality, we have that

$$
\frac{\operatorname{Eng}(G)}{2}=\sum_{i=1}^{p}\left|\mu_{i}\right| \leq \sqrt{p \sum_{i=1}^{p} \mu_{i}^{2}}=\sqrt{p S^{+}} \leq \sqrt{\frac{2 e(\chi-1) p}{\chi}} .
$$

Similarly, we have that

$$
\frac{\operatorname{Eng}(G)}{2}=\sum_{i=n-q+1}^{n}\left|\mu_{i}\right| \leq \sqrt{q \sum_{i=n-q+1}^{n} \mu_{i}^{2}}=\sqrt{q S^{-}} \leq \sqrt{\frac{2 e(\chi-1) q}{\chi}} .
$$

Therefore we get that

$$
\begin{aligned}
& \operatorname{Eng}(G)=\frac{\operatorname{Eng}(G)}{2}+\frac{\operatorname{Eng}(G)}{2} \\
& \leq \sqrt{\frac{2 e(\chi-1) p}{\chi}}+\sqrt{\frac{2 e(\chi-1) q}{\chi}}=\sqrt{\frac{2 e(\chi-1)}{\chi}}(\sqrt{p}+\sqrt{q}) .
\end{aligned}
$$

From Lemma 2.2, we have that $\alpha \leq n-(p+q)+\min \{p, q\} \leq n-p-q+p=n-q$ and $\alpha \leq n-(p+q)+\min \{p, q\} \leq n-p-q+q=n-p$. Thus $p \leq n-\alpha$ and $q \leq n-\alpha$. Therefore we have that

$$
\begin{aligned}
& 2 \sqrt{\frac{2 e(\chi-1)(n-k-1)}{\chi}} \leq \operatorname{Eng}(G) \leq 2 \sqrt{\frac{2 e(\chi-1)(n-\alpha)}{\chi}} \\
& \leq 2 \sqrt{\frac{2 e(\chi-1)(n-s-1)}{\chi}} \leq 2 \sqrt{\frac{2 e(\chi-1)(n-k-1)}{\chi}} .
\end{aligned}
$$

From the above proofs, we have that

$$
S^{+}=S^{-}=\frac{2 e(\chi-1)}{\chi}
$$




$$
\begin{aligned}
& \mu_{1}=\mu_{2}=\cdots=\mu_{p}, \mu_{n-q+1}=\mu_{n-q+2}=\cdots=\mu_{n}, \\
& p=q=n-\alpha, \alpha=s+1=k+1 .
\end{aligned}
$$

Thus $p \mu_{1}^{2}=S^{+}=S^{-}=q \mu_{n}^{2}$. Since $p=q, \mu_{1}^{2}=\mu_{n}^{2}$. Hence $\mu_{1}=-\mu_{n}$. Since $G$ is connected and $\mu_{1}=-\mu_{n}, G$ is a bipartite graph. From Perron-Frobenius theorem, we have that $\mu_{1}>\mu_{2}$. Since $\mu_{1}=\mu_{2}=\cdots=\mu_{p}$, we must have $p=1$. Now $\alpha=n-p=n-1$, which implies that $G$ cannot be 2-connected, a contradiction.

Therefore the proof of Theorem 1 is complete.

Proof of Theorem 1.2. Let $G$ be a graph satisfying the conditions in Theorem 1.2. Suppose, to the contrary, that $G$ is not traceable. If $n=3, G$ must be traceable since $G$ is $k$-connected $(k \geq 1)$. From now on, we assume that $n \geq 4$. Choose a longest path $P$ in $G$ and give an orientation on $P$. Let $x$ and $y$ be the two end vertices of $P$. Since $G$ is not traceable, there exists a vertex $u_{0} \in V(G)-V(P)$. By Menger's theorem, we can find $s(s \geq k)$ pairwise disjoint (except for $\left.u_{0}\right)$ paths $P_{1}, P_{2}, \ldots, P_{s}$ between $u_{0}$ and $V(P)$. Let $v_{i}$ be the end vertex of $P_{i}$ on $P$, where $1 \leq i \leq s$. Without loss of generality, we assume that the appearance of $v_{1}, v_{2}, \ldots, v_{s}$ agrees with the orientation of $P$. Since $P$ is a longest path in $G, x \neq v_{i}$ and $y \neq v_{i}$, for each $i$ with $1 \leq i \leq s$, otherwise $G$ would have paths which are longer than $P$. We use $v_{i}^{+}$to denote the successor of $v_{i}$ along the orientation of $P$, where $1 \leq i \leq s$. Since $P$ is a longest path in $G$, we have that $v_{i}^{+} \neq v_{i+1}$, where $1 \leq i \leq s-1$. Moreover, $S:=\left\{u_{0}, v_{1}^{+}, v_{2}^{+}, \ldots, v_{s}^{+}, x\right\}$ is independent (otherwise $G$ would have paths which are longer than $P$ ). Then $\alpha \geq s+2 \geq k+2$.

Using the arguments similar to the ones in Proof of Theorem 1.1, we have that

$$
\begin{aligned}
& 2 \sqrt{\frac{2 e(\chi-1)(n-k-2)}{\chi}} \leq \operatorname{Eng}(G) \leq 2 \sqrt{\frac{2 e(\chi-1)(n-\alpha)}{\chi}} \\
& \leq 2 \sqrt{\frac{2 e(\chi-1)(n-s-2)}{\chi}} \leq 2 \sqrt{\frac{2 e(\chi-1)(n-k-2)}{\chi}} .
\end{aligned}
$$

Therefore we have that

$$
\begin{aligned}
& S^{+}=S^{-}=\frac{2 e(\chi-1)}{\chi} \\
& \mu_{1}=\mu_{2}=\cdots=\mu_{p}, \mu_{n-q+1}=\mu_{n-q+2}=\cdots=\mu_{n}, p=q=n-\alpha, \alpha=s+2=k+2 .
\end{aligned}
$$

Thus $p \mu_{1}^{2}=S^{+}=S^{-}=q \mu_{n}^{2}$. Since $p=q, \mu_{1}^{2}=\mu_{n}^{2}$. Hence $\mu_{1}=-\mu_{n}$. Since $G$ is connected and $\mu_{1}=-\mu_{n}$, $G$ is a bipartite graph. From Perron-Frobenius theorem, we have that $\mu_{1}>\mu_{2}$. Since $\mu_{1}=\mu_{2}=\cdots=\mu_{p}$, we must have $p=1$. Now $\alpha=n-p=n-1$. So $G$ is $K_{1, n-1}$ with $n \geq 4$. Since now $k=1$ and $n-1=\alpha=k+2$, we have that $G$ is $K_{1,3}$.

Therefore the proof of Theorem 1.2 is complete.

\section{References}

[1] J. A. Bondy, U. S. R. Murty, Graph Theory with Applications, The Macmillan Press LTD, 1976.

[2] I. Gutman, The energy of a graph, Berichte der Mathematisch-Statistischen Sektion im Forschungszentrum Graz, 103 (1978), $1-12$.

[3] T. Ando, M. Lin, Proof of a conjectured lower bound on the chromatic number of a graph, Linear Algebra Appl., 485 (2015), $480-484$.

[4] D. Cvetković, M. Doob, H. Sachs, Spectra of Graphs - Theory and Application, 3rd Edition, Johann Ambrosius Barth, 1995.

[5] R. Li, A sharp upper bound for the energy of a connected graph, Manuscript, July 2018. 